\title{
Effect of intermixed foveal and parafoveal presentation on same-different judgments: Evidence for a criterion-inertia model
}

\author{
LESTER E. KRUEGER \\ Ohio State University, Columbus, Ohio
}

\begin{abstract}
Subjects judged whether two adjacent letters, which were presented either $0.5^{\circ}$ (foveal or near condition) or $2.0^{\circ}$ (parafoveal or far condition) from fixation, were identical or different. The preponderance of false-different responses (i.e., errors on same trials) over false-same ones increased, whereas the fast-same effect was eliminated, on the far pairs, but only when they were intermixed with near pairs rather than presented in separate (pure) blocks of trials. Intermixing the near and far pairs produced the opposite trends on near trials (i.e., smaller preponderance of false-different errors, larger fast-same effect). Carr et al., who presented trigrams, found a similar criterion misadjustment, which likewise depended on intermixed presentation. They proposed that their easy (familiar or orthographically regular) pairs produced a bias or criterion shift towards "same." No obvious biasing features were present in the near or far pairs, however, so the criterion misadjustment found here was attributed to the combined effect of internal noise and criterion inertia, not to criterion shifting. Increasing the level of internal noise on far trials produced more spurious perceived mismatches, but on mixed blocks of near and far trials, subjects relied on a common, compromise criterion for responding versus rechecking (recheck moderate perceived differences), instead of the separate, more appropriate criteria for near trials (recheck small differences) and far trials (recheck large differences) used on pure blocks.
\end{abstract}

When people judge whether two letters are identical or not, same judgments typically are faster than different judgments. According to the noisy-operator theory (Krueger, 1978), internal noise more often changes physical matches into spurious perceived mismatches than vice versa. As a result, different judgments are slower, owing to more rechecking of perceived mismatches, and there typically is a tendency to make more false-different responses (i.e., errors on same trials) than false-same ones.

If internal noise is so important a factor, then the fastsame effect ought generally to be quite large under difficult or demanding conditions (Krueger \& Gott, in press). However, the fast-same effect typically is reduced or reversed when the elements are difficult to encode or discriminate (Bindra, Donderi, \& Nishisato, 1968; Nickerson, 1972) or when less familiar stimuli are presented (Egeth \& Blecker, 1971; see Krueger's, 1975, review, p. 960). The reduction in the fast-same effect might be due to a bias towards "same" on easy pairs or a bias towards "different" on difficult pairs (Carr, Posner, Pollatsek, \& Snyder, 1979), because errors tend to increase more on same than on different trials under difficult conditions (Carr et al., 1979; Krueger \& Gott, in press). Al-

The author is grateful to Mark H. Chignell for helpful discussions, and to Ronald G. Shapiro and Robert W. Proctor for useful comments on an earlier version of this report. Requests for reprints should be sent to Lester E. Krueger, Human Performance Center, Ohio State University, 404-B West 17th Avenue, Columbus, OH 43210. ternatively, the seeming criterion misadjustment (reduced fast-same effect, increased preponderance of falsedifferent errors) on difficult (vs. easy) trials may be due not to criterion shifting, but to its opposite, criterion inertia. What really changes under difficult conditions may be the number of spurious matches perceived, owing to the increased level of internal noise. The present paper examines not only whether the misadjustment involves response bias or criterion inertia (Question 1), but also whether it is greater when difficult and easy trials are intermixed rather than blocked separately (Question 2). If the misadjustment involves the criterion, rather than some other process such as trace strength, then it should be greater when difficult and easy trials are intermixed. Intermixing would exacerbate the misadjustment of the criterion, either because it highlights or makes salient those features producing the bias towards "same" or "different" (response-bias model), or because it forces the subject to make rapid adjustments of the criterion to varying levels of internal noise (criterion-inertia model).

\section{Question 1}

Does criterion misadjustment on difficult (vs. easy) trials involve response bias or criterion inertia? A misadjustment may arise either because subjects shift the criterion (owing to response bias) when they should not, or because they fail to shift it (owing to criterion inertia) when they should. Carr et al.(1979) varied the familiarity and orthography of trigrams, and they found a smaller fast-same effect and a greater proportion of false-different 
errors on unfamiliar or irregular trigrams. They concluded that their subjects "were strongly biased towards responding same by the occurrence of anything either regular or familiar" (p. 396). Biasing features of various sorts might indeed affect same-different judgments. Thus, responses may be slower on similar than on dissimilar different pairs owing to response competition induced by matching features (Chignell \& Krueger, 1984; Eriksen, O’Hara, \& Eriksen, 1982). Chignell and Krueger found that falsesame responses on similar different pairs exceeded those on dissimilar different pairs. However, they also found that false-different responses exceeded false-same responses even on similar different pairs, which indicates that the response-competition model must be supplemented by the internal-noise principle (Krueger, 1978) in order to explain the fast-same effect.

One limitation of the response-bias model in explaining criterion misadjustment is that it fails to take account of changes in the error tendency owing to increased internal noise under difficult conditions. Interestingly, subjects also may suffer from this limitation. Thus, the criterion-inertia model attributes criterion misadjustment under difficult conditions to subjects' failure to allow for an increase in spurious mismatches. Subjects might tend to rely entirely on the criterion appropriate on easy trials. Figure 1 (adapted from Krueger, 1978, Figure A1) shows

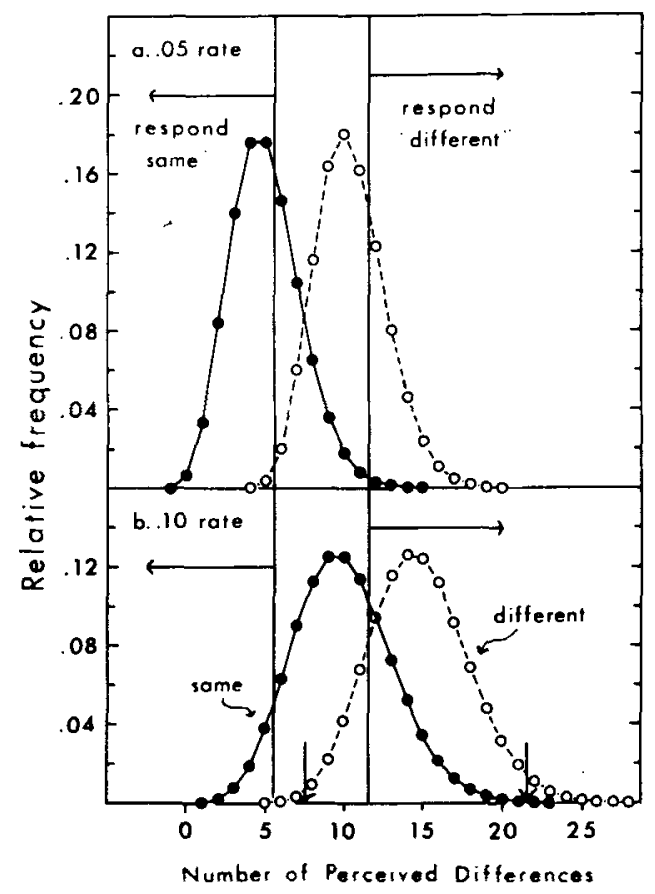

Figure 1. Predicted number of perceived featural mismatches on Pass 1, given that the probability of feature failure is .05 (Panel a) or $\mathbf{1 0}$ (Panel b), and that the number of physical mismatches out of 100 is 0 (same) or 6 (different). The criterion lines appropriate to the .05 rate in Panel a were extended downward in Panel $b$ to illustrate criterion inertia; the two vertical arrows in Panel b show the appropriate criterion adjustment to the $\mathbf{. 1 0}$ rate. (Adapted from Krueger, 1978, Figure A1.) what would happen, according to the noisy-operator theory, if the criterion were not adjusted upward when the risk of feature failure (reversal in match/mismatch status) increased from $5 \%$ (Figure 1a) to $10 \%$ (Figure $1 \mathrm{~b}$ ). In Figure 1a, rechecking is properly directed to those perceived difference counts for which the risk of error on an immediate response exceeds $4 \%$. (The $4 \%$ risk reflects the 25-to-1 likelihood ratio seemingly used as a decision rule in studies whose data were fitted by Krueger, 1978.) Internal noise tends to increase, but not decrease, the perceived difference count, thus producing a positive skew. As a result, the same distribution undermines the different distribution and many more different pairs are rechecked (fast-same effect), whereas more same than different pairs fall into the immediate-response region of the opposite trial type (preponderance of false-different errors). Figure $1 \mathrm{~b}$ shows that retaining the same rechecking region as the level of internal noise increased (i.e., criterion inertia) would eliminate or reverse the fast-same effect, producing far more immediate different than same responses, as well as an even greater preponderance of false-different responses. (The two downward arrows in Figure $1 \mathrm{~b}$ show the appropriate criterion adjustment.) Another possibility is that the common rechecking region would occupy an intermediate, compromise position between the settings appropriate for easy and difficult trials. This, too, would produce the characteristic criterion misadjustment (reduced fast-same effect, increased proportion of false-different errors) evident on difficult (vs. easy) trials.

To show that such criterion inertia can occur, criterion misadjustment must be found when no obvious biasing features are present on difficult or easy trials. In detection tasks, subjects typically are conservative and resist changing the criterion fully when relative frequency or payoffs are changed (Green \& Swets, 1966). In the samedifferent judgment task, likewise, criterion stability or inertia, not change, likely is the norm and thus ought to be presupposed when no obvious biasing features or imbalances, for example, in the relative frequency of same vs. different trials (Ratcliff, 1985), are present. Krueger and Shapiro (1985) varied the interstimulus interval (ISI) between two visual patterns from .25 to $3 \mathrm{sec}$, which introduced no obvious biasing features, yet they found criterion misadjustment on difficult (vs. easy) trials. With a long ISI, the fast-same effect was eliminated or reversed, whereas false-different errors abounded. Fitting the criterion-inertia model to their data, they estimated that $55 \%$ of subjects' decisions were based on a common criterion and $45 \%$ on the criterion appropriate for the particular level of ISI used on the trial. The present study provided a further test of the criterion-inertia model, since there were no obvious biasing features in the letter pairs presented near $\left(0.5^{\circ}\right)$ or far $\left(2.0^{\circ}\right)$ from fixation.

\section{Question 2}

Does intermixing difficult and easy trials increase the criterion misadjustment on difficult (vs. easy) trials? Carr 
et al. (1979) found such an intermixing effect, but Krueger and Shapiro (1985) did not. Carr et al. obtained a smaller fast-same effect and a greater proportion of falsedifferent errors on unfamiliar or irregular trigrams when these were intermixed with familiar or regular ones, but not when they were blocked separately. They concluded that "random versus blocked stimulus presentation is a methodological variable that can exercise considerable control over the setting of response criteria in matching studies of word processing"' (p. 398). Krueger and Shapiro, by contrast, found criterion misadjustment on long-ISI trials which was equally strong with blocked and intermixed presentation. It is not clear why blocking did not reduce or eliminate the misadjustment. Perhaps decay in the memory trace of the first pattern with a long ISI not only prevented rechecking, but also somehow inhibited proper adjustment of the criterion. Alternatively, what appears to be misadjustment of the criterion may actually have involved another process, such as memory trace strength.

In the present study, the memory factor was excluded by the presentation of two letters simultaneously for comparison, either near $\left(0.5^{\circ}\right)$ or far $\left(2.0^{\circ}\right)$ from fixation. Blocking was varied, because previous findings had suggested that criterion misadjustment on far (vs. near) pairs would be found only when far and near trials were intermixed, but not when they were blocked separately. Krueger and Gott (in press) presented letter pairs that were uniformly quite difficult $\left(1.58^{\circ}\right.$ from fixation; brief, laterally masked presentation), which apparently allowed subjects to properly adjust the overall criterion, because they found a large, nearly 70 -msec fast-same effect and considerably more false-different than false-same errors. The fast-same effect did not increase further, however, on those intermixed letter pairs that were made even more difficult (mirror-reversed letter orientation; outer or more peripheral lateral mask) than the rest. Similarly, criterion misadjustment was evident when near and far pairs were intermixed in other studies; both RT and errors increased more on same (vs. different) pairs, with false-same errors actually declining, as the distance from fixation increased from $0^{\circ}$ to $4^{\circ}$ (Fisher \& Lefton, 1976, Experiment 3; Lefton \& Haber, 1974, Experiment 1). In an oddity-detection task, Millspaugh (1978) intermixed displays that varied in the mean element distance from the center, and he found a smaller fast-same effect, along with more false-different errors, on the more peripheral displays.

In the present study, the near and far pairs were intermixed on some (mixed) blocks of trials, but presented separately on other (pure) blocks. It was expected that subjects would tend to use a common, compromise criterion for near and far trials on the mixed blocks, but not on the pure blocks. On mixed blocks, the criterion might not remain stationary at an intermediate position, but fluctuate between settings appropriate for near and far trials. Considerable adjustment of the criterion might occur after, even if not during, each trial. If so, then the difficulty of the immediately preceding trial ought to have a considerable effect, which will be examined in a special, sequential analysis of mixed-block data. After a near trial, a subject would expect less of an effect of internal noise, and the criterion would be adjusted downward (i.e., the rechecking region would be moved leftward in Figures $1 \mathrm{a}$ and $1 \mathrm{~b}$ ), thus decreasing the fast-same effect and increasing false-different errors. After a far trial, by contrast, the subject would anticipate more of an effect of internal noise and the criterion would be adjusted upward, thus increasing the fast-same effect and decreasing falsedifferent errors.

\section{METHOD}

\section{Subjects}

Twenty Ohio State University undergraduates participated in order to receive credit in an introductory psychology course. All subjects had at least 20/30 (corrected) vision, as tested with a Snellen chart, and all made fewer than $10 \%$ errors. Data were excluded on nine additional subjects, who reported after the session that they had frequently looked far to the left or right, rather than at the fixation mark as instructed; their errors were about $10 \%$ or higher.

\section{Apparatus}

Uppercase letters were presented at a $60-\mathrm{Hz}$ refresh rate and at 32-cd $/ \mathrm{m}^{2}$ intensity on a greenish-tint, fast-decay P31 phosphor (decay to $1 \%$ intensity at .25 msec after display offset) by an Imlac PDS-4 graphics computer, which also measured RT to an accuracy of $1 \mathrm{msec}$. The letters, presented as thin, illuminated lines on a dark screen, were software generated, using short line vectors, so as to resemble normal English uppercase letters. Each subject sat alone in a dark room, with the head held fast in a chinrest located $70 \mathrm{~cm}$ from the display screen.

\section{Stimulus Materials}

The letters were randomly selected from the set $F, G, J, K, L$, which are about equally discriminable from each other. Each letter was $0.29 \mathrm{~cm}$ wide and $0.43 \mathrm{~cm}$ high, and $0.40 \mathrm{~cm}$ separated the two letters vertically. The pair thus was $1.26 \mathrm{~cm}\left(1.03^{\circ}\right)$ high, and it was centered at the same height and located $0.61 \mathrm{~cm}\left(0.5^{\circ}\right)$ or $2.44 \mathrm{~cm}\left(2.0^{\circ}\right)$ to the left or right of the fixation mark.

Each block of 144 trials contained 36 replicas of all combinations of the following factors: visual field of letter pair (left, right) and trial type (same, different) $(36 \times 2 \times 2=144)$. One block of trials consisted of near pairs ( $0.5^{\circ}$ from fixation mark), a second block consisted of far pairs ( $2.0^{\circ}$ from fixation mark), and the other two blocks were mixed (i.e., 72 near pairs and 72 far pairs). The two mixed blocks were presented contiguously, either before (10 subjects) or after (10 subjects) the two pure blocks. Ten subjects received the near block before the far block, and the other 10 had the reverse order. Four different randomized sequences of letter pairs and trials within blocks were used.

Each block consisted of four subblocks of 36 trials each. There was a 15-sec interval between subblocks, which allowed the next set of trials to be read in from the host computer. Each subblock was preceded by four practice trials, and there was a practice subblock (mixed distances) preceding the four regular blocks, for a total of 17 subblocks, or 680 trials (576 regular, 104 practice) in all.

\section{Procedure}

Each trial began with a .6-sec presentation of the fixation mark alone. The letter pair then appeared for $100 \mathrm{msec}$, followed by the fixation mark alone again until the subject responded. Feedback on response accuracy appeared in the upper part of the screen, directly above the fixation mark, during the 1.5 -sec interval be- 
tween trials. The subjects were instructed to keep their eyes focused at all times on the fixation mark, and to respond as rapidly as possible, but not at the expense of accuracy. They were cautioned that the letter pair would appear too briefly to let them move their eyes to focus on it.

Ten subjects pressed a right-hand button for same judgments and a left-hand button for different judgments, and the other ten had the reverse hand assignment. Trials with an RT greater than $3 \mathrm{sec}$ or less than $200 \mathrm{msec}$ were discarded prior to data analysis. Mean RT was computed for correct trials only.

\section{RESULTS and DISCUSSION}

As shown in Figure 2, the fast-same effect was present, but only marginally significant, $\mathrm{F}(1,19)=3.61, \mathrm{p}<.10$, whereas the preponderance of false-different errors was highly significant, $F(1,19)=23.66, p<.001$. As expected, far trials were more difficult than near trials, producing both longer RT, $F(1,19)=43.40, \mathrm{p}<.001$, and greater errors, $F(1,19)=58.80, p<.001$. Several subjects said afterwards that they could more readily focus their attention on two potential locations (pure blocks) than on four (mixed blocks), but the somewhat lower RT on pure (vs. mixed) blocks was not significant, $F(1,19)$ $=1.88$, and errors did not differ, $\mathrm{F}<1$.

Distance from fixation interacted significantly with trial type (same vs. different) on both RT $[\mathrm{F}(1,19)=8.86$, $\mathrm{p}<.01]$ and errors $[\mathrm{F}(1,19)=15.22, \mathrm{p}<.001]$. The fast-same effect decreased, whereas the preponderance of false-different errors increased, on far (vs. near) trials (see Figure 2). Thus, criterion misadjustment was evident on difficult (vs. easy) trials, confirming similar findings by Carr et al. (1979), Fisher and Lefton (1976), Krueger and Shapiro (1985), and Lefton and Haber (1974). The fast-

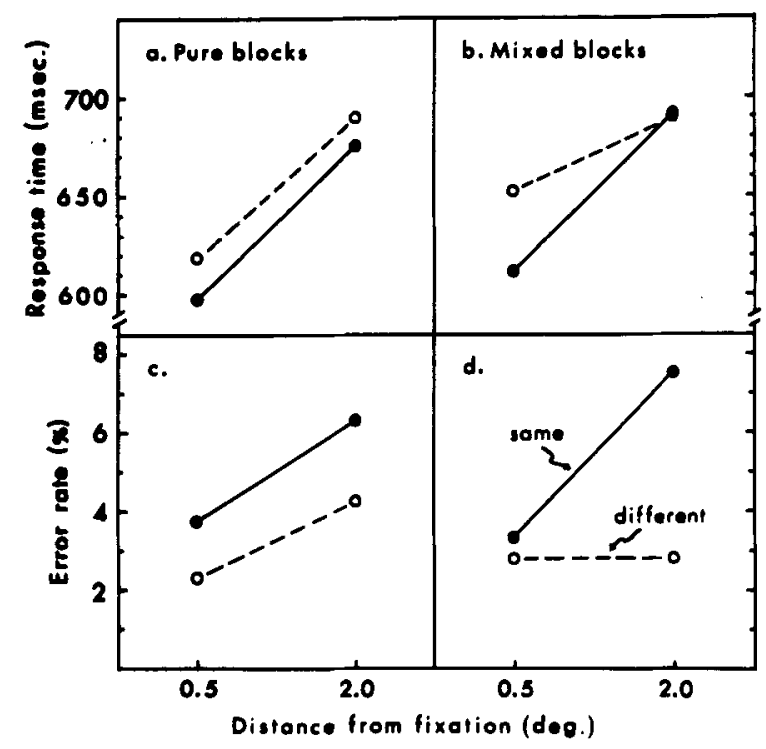

Figure 2. Mean response time and percentage of error rate by trial type (same, different), distance of letter pair from fixation $\left(0.5^{\circ}, 2.0^{\circ}\right.$ ), and block type (pure, mixed). same effect was significant on near trials analyzed separately $[F(1,19)=10.24, p<.01]$ but not on far trials $(\mathrm{F}<1)$, whereas the preponderance of false-different $\mathrm{er}-$ rors was significant on both near trials $[F(1,19)=9.81$, $\mathrm{p}<.01]$ and far trials $[\mathrm{F}(1,19)=23.31, \mathrm{p}<.001]$.

Furthermore, the intermixing effect (Carr et al., 1979) was obtained. The disparity between near and far trials was much more prominent on mixed (vs. pure) blocks (see Figure 2). The distance $\times$ trial type $\times$ pure vs. mixed interaction was significant on both $\mathrm{RT}[\mathrm{F}(1,19)=5.17$, $p<.05]$ and errors $[F(1,19)=4.70, p<.05]$. Some criterion misadjustment was evident even on pure far trials (note the convergence on same vs. different RT on far trials in Figure $2 \mathrm{a}$ and the divergence on errors in Figure 2c) but it was very slight. On the pure blocks analyzed separately, the distance $\times$ trial type interaction was significant on neither $\mathrm{RT}$ nor errors ( $\mathrm{F}<1$ in both cases), whereas on the mixed blocks, it was quite significant on both RT $[F(1,19)=12.21, \mathrm{p}<.01]$ and errors $[F(1,19)$ $=16.17, \mathrm{p}<.001]$.

Thus, the subjects were able to adjust the criterion appropriately, or nearly so, on near and far trials in the pure blocks. Since feedback on the accuracy of the response was given after every trial, subjects knew when they made a false-different response and thus had the information needed to properly adjust the criterion. Several subjects said afterwards that they figured out that if the two letters looked similar enough (e.g., J and I; E and F), they really were identical. Krueger and Shapiro also provided feedback on response accuracy, but found no effect of blocking vs. intermixing, apparently because of memory factors introduced by their ISI variable.

On mixed blocks, by contrast, the subjects did not adjust the criterion separately for near and far trials, but relied on a common criterion, and as a result, both RT (Figure 2b) and errors (Figure 2d) increased more on same (vs.different) pairs as the distance from fixation increased, just as Fisher and Lefton (1976) and Lefton and Haber (1974) had found. A comparison with the pure blocks shows that the criterion misadjustment occurred on both near and far pairs in the mixed blocks. Thus, in going from pure to mixed blocks, the fast-same effect increased on near trials from 21 to $41 \mathrm{msec}$, but decreased on far trials from 14 to $-2 \mathrm{msec}$; the preponderance of false-different errors decreased on near trials from $1.47 \%$ to $.52 \%$, but increased on far trials from $2.03 \%$ to $4.72 \%$. These shifts indicate that subjects did not simply revert to the criterion appropriate for near trials (see Figures 1a and $1 \mathrm{~b}$ ), but used a compromise criterion situated on the average about midway between the criteria appropriate for near and far trials.

\section{Visual Field}

Mean RT was slightly, but not significantly, lower for the left- than for the right-field pairs [650 vs. $658 \mathrm{msec}$; $\mathrm{F}(1,19)=1.16]$, and the same was true on errors $[3.72 \%$ vs. $4.53 \% ; F(1,19)=2.01]$. The subjects apparently saw 
the letters slightly more clearly in the left visual field, but relied on a common criterion for the two fields, because visual field interacted significantly with trial type on both $\operatorname{RT}[F(1,19)=5.54, p<.05]$ and errors $[F(1,19)$ $=4.93, \mathrm{p}<.05]$. The fast-same effect was larger for the left- than for the right-field pairs ( $34 \mathrm{vs.} 3 \mathrm{msec}$ ), whereas the preponderance of false-different errors was smaller $(1.16 \%$ vs. $3.21 \%)$. Thus, the same criterion misadjustment on difficult (vs. easy) trials was evident on both left vs. right trials and near vs. far trials. On left vs. right, as on near vs. far, there were no obvious biasing features that would prompt a person to respond "same" on an easy (i.e., left) pair or "different" on a difficult (i.e., right) pair, so the misadjustment must have been produced by criterion inertia rather than response bias.

The slight left-field advantage probably was not due to leftward eye movements. After the session, subjects reported looking slightly to the left of fixation on $19 \%$ of the trials, slightly to the right on $20 \%$, and directly at the fixation mark on $61 \%$. The uppercase letters used here may have been slightly more visible or discriminable in the left visual field because they opened mainly towards the right $(F, G, K, L)$, rather than towards the left (J).

\section{Pure Blocks}

A second analysis was restricted to trials on pure blocks. Each pure block consisted of four successive subblocks of 36 trials each, and the subblock factor was added in this analysis to determine whether there was a progressive adjustment or shift in the criterion across subblocks. However, neither the main effect of subblock nor its interaction with distance $\times$ trial type was significant, on either RT or errors. On far trials, the fast-same effect was $-10,1,3$, and $27 \mathrm{msec}$, respectively, for Subblocks 1, 2,3 , and 4 , whereas on near trials it was $45,9,35$, and $6 \mathrm{msec}$, respectively; the preponderance of false-different errors on far trials was $0.67 \%, 3.68 \%, 1.19 \%$, and $2.52 \%$, respectively; on near trials, it was $4.17 \%, 1.95 \%$, $-0.60 \%$, and $0.56 \%$, respectively. The increased internal noise on far blocks was expected to increase the speed advantage for same trials. Krueger and Gott (in press) found a large, nearly 70-msec fast-same effect when trials were uniformly quite difficult (brief, parafoveal, laterally masked presentation). However, there was little tendency here for the fast-same effect to increase across the four subblocks on far blocks, and even on the fourth subblock, it attained a value of only $27 \mathrm{msec}$. Perhaps the switching between mixed and pure near and pure far blocks, as well as exposure to the initial mixed practice subblock, prompted subjects to rely somewhat on a common, compromise criterion for the session as a whole. However, the use of a lateral mask, not the uniform high difficulty of the trials, may have produced the large fastsame effect Krueger and Gott found.

\section{Mixed Blocks}

Subblock level in a pure block may have had no effect because the criterion shifted early in the first subblock.
Even a single trial might greatly shift the criterion. A third analysis, which was restricted to trials on mixed blocks, examined the effect of the preceding trial. When the preceding trial was near rather than far, the fast-same effect was slightly, but not significantly, smaller (15 vs. $22 \mathrm{msec} ; \mathrm{F}<1$ ), whereas the preponderance of falsedifferent errors was significantly larger $[3.74 \%$ vs. $1.70 \% ; F(1,19)=5.51, p<.05]$. After a near trial, subjects apparently expected less of an effect of internal noise, and adjusted the criterion downward accordingly, thus decreasing the fast-same effect, though not significantly, and increasing false-different errors. After a far trial, the reverse occurred.

\section{CONCLUSIONS}

Criterion misadjustment (reduced fast-same effect, increased preponderance of false-different errors) was found on difficult (far or right) trials, but only when they were intermixed with easy (near or left) trials. Carr et al. (1979), who found similar results with trigrams, attributed the misadjustment to response bias; that is, familiar or regular pairs tend to evoke the "same" response, whereas unfamiliar or irregular pairs tend to evoke the "different" response. Response bias cannot explain the present results, however, because there were no obvious biasing features present that would tend to evoke the "same" response on near or left pairs, or the "different" response on far or right pairs. Furthermore, the biasing features would have had to be equally strong on near and far trials, which were affected to the same extent, but in opposite direction, by intermixing (see Figure 2). The criterioninertia model would explain this symmetry more parsimoniously as simply due to the use of a compromise criterion. Response bias may be induced by varying the proportion or relative frequency of same and different trials (Ratcliff, 1985), but there was no variation in relative frequency or payoffs in the present study. Krueger and Shapiro (1985) also provided no obvious biasing features, and they, too, found misadjustment on difficult (long-ISI) trials. Their effect may not have involved the criterion, though, since they failed to find the intermixing effect.

The present results do not rule out the response-bias factor, which may be important in some cases (Ratcliff, 1985). In fitting tone-comparison data from Bindra, Williams, and Wise (1965), for example, Krueger (1979, Case 1) had to allow for a large response bias towards "different," in addition to external noise (heterogeneity of difference), in order to account for a large fast-different effect and a large preponderance of false-different errors. However, people often resist changing the criterion (Green \& Swets, 1966), and misadjustments may arise more frequently from criterion inertia than from criterion shifting.

It should be noted, though, that the response-bias and criterion-inertia models are yet very similar. They agree on the basic phenomenon of criterion misadjustment. They disagree on whether the misadjustment is due to the com- 
bined effect of internal noise and criterion inertia, or simply to the effect of biasing features in easy or difficult pairs. Biasing features may not always be obvious, and, if so, one need not postulate imaginary or hypothetical biasing features, where none might really exist, but need merely allow for the effect of internal noise. The responsebias model makes the dubious assumption that increasing the task difficulty would increase the error tendency equally on same and different trials. Perhaps the key advantage of the criterion-inertia model is that it takes into account and makes use of, rather than ignores, the tendency for increased internal noise to produce more spurious perceived mismatches.

\section{REFERENCES}

BindRa, D., Donderi, D. C., \& Nishisato, S. (1968). Decision latencies of "same" and "different" judgments. Perception \& Psychophysics, 3, 121-130.

Bindra, D., Willans, J. A., \& WiSe, J. S. (1965). Judgments of sameness and difference: Experiments on decision tiime. Science, 150, 1625-1627. (Erratum, p. 1699)

Carr, T. H., Posner, M. I., Pollatsek, A., \& Snyder, C. R. R. (1979). Orthography and familiarity effects in word processing. Journal of Experimental Psychology: General, 108, 389-414.

Chignell, M. H., \& KRUEger, L. E. (1984). Further evidence for priming in perceptual matching: Temporal, not spatial, separation enhances the fast-same effect. Perception \& Psychophysics, 36, 257-265.

EGETH, H., \& BLECKER, D. (1971). Differential effects of familiarity on judgments of sameness and difference. Perception \& Psychophysics, 9, 321-326.

Eriksen, C. W., O'Hara, W. P., \& Eriksen, B. A. (1982). Response competition effects in same-different judgments. Perception \& Psychophysics, 32, 261-270.

Fisher, D. F., \& Lefton, L. A. (1976). Peripheral information extraction: A developmental examination of reading processes. Journal of Experimental Child Psychology, 21, 77-93.

GREeN, D. M., \& SwETs, J. A. (1966). Signal detection theory and psychophysics. New York: Wiley.

KRUEGER, L. E. (1975). Familiarity effects in visual information processing. Psychological Bulletin, 82, 949-974.

Krueger, L. E. (1978). A theory of perceptual matching. Psychological Review, 85, 278-304.

KRUEGER, L. E. (1979). A model of unidimensional perceptual matching. Journal of Experimental Psychology: Human Perception and Performance, 5, 277-288.

Krueger, L. E., \& GotT, R. E. (in press). Effect of lateral masking and letter reversal on same-different judgments. Bulletin of the Psychonomic Society.

Krueger, L. E., \& Shapiro, R. G. (1985). Effect of interstimulus interval and heterogeneity of difference on same-different judgments of visual patterns. Bulletin of the Psychonomic Society, 23, 43-46.

LEFTON, L. A., \& HABER, R. N. (1974). Information extraction from different retinal locations. Journal of Experimental Psychology, 102, 975-980.

MillsPaUGH, J. R. (1978). Effects of array organization on samedifferent judgments. Perception \& Psychophysics, 23, 27-35.

Nickerson, R. S. (1972). Auditory codability and the short-term retention of visual information. Journal of Experimental Psychology, 95, 429-436.

RATCLIFF, R. (1985). Theoretical interpretations of the speed and accuracy of positive and negative responses. Psychological Review, 92, 212-225.

(Manuscript received December 7, 1984; revision accepted for publication February 23, 1985.) 